\title{
Análise temporal da qualidade da água do Rio Tramandaí/RS
}

A qualidade da água está intimamente relacionada as suas características físicas, químicas e biológicas e estas são função dos diferentes usos consuntivos dos mananciais. Cientes disto este trabalho objetivou identificar as possíveis fontes de poluição do rio Tramandaí/RS. Para isto foram utilizados dados secundários de qualidade da água, disponibilizados pela Fundação Estadual de Proteção Ambiental Henrique Luis Roessler. Para isto foi aplicada a análise fatorial seguida da análise de componentes principais. Como resultado foi possível observar que as variáveis $\mathrm{CE}, \mathrm{ST}, \mathrm{TH}, \mathrm{OD}$, TH2O, TAR e ST da água apresentaram maiores coeficientes de correlação. E com a decomposição da matriz de correlação obteve-se a formação de duas componentes principais, através das quais foi possível identificar as principais possíveis fontes de poluição. Através deste estudo foi possível concluir a analise fatorial/ análise de componentes principais são ferramentas que pode ser utilizada para fins de monitoramento da qualidade da água quando se busca investimentos de baixo custo com resultados confiáveis e rápidos.

Palavras-chave: Análise Fatorial; Análise de Componentes Principais; Fontes de Poluição.

\section{Temporal analysis of the water quality of the Tramandai River/RS}

\begin{abstract}
The quality of water is closely related to its physical, chemical and biological characteristics and these are a function of the different consumptive uses of the water sources. Aware of this, this work aimed to identify the possible sources of pollution of the Rio Tramandai / RS. For this purpose, secondary data on water quality were used, provided by the State Foundation for Environmental Protection Henrique Luis Roessler. For this purpose, factorial analysis followed by principal component analysis was applied. As a result, it was possible to observe that the variables EC, TS, TH, DO, H2OT, ART and TS of the water had higher correlation coefficients. And with the decomposition of the correlation matrix the formation of two main components was obtained, through which it was possible to identify the main possible sources of pollution. Through this study it was possible to conclude the factorial analysis / analysis of main components are tools that can be used for water quality monitoring purposes when searching for low cost investments with reliable and fast results.
\end{abstract}

Keywords: Factor Analysis; Principal Component Analysis; Pollution Sources.

Topic: Engenharia de Recursos Hídricos

Reviewed anonymously in the process of blind peer.
Received: 10/06/2018

Approved: 24/07/2018
Luana Nunes Centeno

Universidade Federal de Pelotas, Brasil http://orcid.org/0000-0001-9398-9863

luananunescenteno@gmail.com

Samanta Tolentino Cecconello (iD)

Instituto Federal Sul-Rio-Grandense, Brasil

http://lattes.cnpq.br/0667595394126577

http://orcid.org/0000-0001-5903-6241

satolentino@gmail.com
Referencing this:

CENTENO, L. N.; CECCONELLO, S. T.. Análise temporal da qualidade da água do Rio Tramandaí/RS. Revista lbero Americana de Ciências Ambientais, v.9, n.5, p.99-105, 2018. DOI: http://doi.org/10.6008/CBPC2179-6858.2018.005.0010 


\section{INTRODUÇÃO}

As características intrínsecas e extrínsecas dos corpos d'água estão relacionas em grande parte aos usos que estas águas se destinam. Dentre os usos consuntivos pode-se citar o abastecimento doméstico e industrial, e as atividades agropecuárias, que se expandem cada vez mais, devido ao crescimento populacional e urbano. A aplicação em grande escala de fertilizantes e a utilização de pesticidas e inseticidas para a produção agrícola vêm aumentando potencialmente os cenários de poluição nos cursos d'água (VOZA et al., 2015).

Além disso, segundo Monica et al. (2016), esses usos consuntivos desordenados podem causar efeitos não só no ecossistema aquático, mas também à saúde humana, pois o corpo hídrico é o resultado das atividades existentes em uma bacia hidrográfica. O lançamento desordenado de esgoto sanitário é considerado um dos principais causadores de poluição nos corpos hídricos, uma vez que é rico em matéria orgânica, e quando lançado in natura, dependendo da capacidade do manancial em se autodepurar, pode resultar em sérios impactos à qualidade da água, como toxicidade e proliferação de algas (HELLER et al., 2010; BERTOSSI et al., 2013; VAROL et al., 2014).

Frente a isto faz-se necessário o monitoramento dos corpos hídricos, e como na maioria das vezes esse monitoramento demanda de acentuados investimentos é indispensável a avaliação e seleção dos principais parâmetros de qualidade da água. Atualmente para a seleção destes parâmetros bem como para identificação das possíveis fontes de poluição tem se utilizado a estatística multivariada (GOMES et al., 2014; HASHEMI et al., 2014; MONICA et al., 2016).

\section{REVISÃO TEÓRICA}

\section{Qualidade da água}

\section{Estatística Multivariada}

Dentre as técnicas empregadas encontram-se a análise fatorial como forma de identificar os fatores e a análise de componentes principais (ACP) como método de extração e identificação destes fatores (BODRUD-DOZA et al., 2016). Os primeiros conceitos da AF surgiram em 1888 por Galton, quando apresentou os métodos de regressão e o coeficiente de correlação (FERREIRA, 2010). Posteriormente, buscando entender as causas que relacionava a inteligência humana e as notas (denominadas posteriormente de escores), obtidas em algumas disciplinas, Charles Spearman, em 1904, criou a atual modelagem da estrutura fatorial, com apenas um fator (CORRAR et al., 2014).

Já a denominação de AF foi disseminada em 1931 por Louis L. Thurstone, que passou a analisar múltiplos fatores e, desde então, tem sido conhecida como uma técnica de interdependência, a partir da qual busca-se elucidar estruturas pertinentes entre as variáveis da análise (VICINI et al., 2005). Essa técnica fornece meios de analisar a estrutura das correlações de uma amostra, com inúmeras variáveis, tornando assim, conhecidos os conjuntos de variáveis altamente correlacionadas, que são denominados de fatores, sendo esses tratados como representantes de dimensões dentro dos dados (HAIR et al., 2009). 
Por conseguinte, para extração destes fatores conforme supracitado pode fazer-se uso da ACP, que almejará uma combinação linear entre as variáveis originais, buscando que o máximo de variância seja explicado por essa combinação (BADILLO-CAMACHO et al., 2015). Posteriormente, retira-se a variância já explicada e busca-se uma nova combinação linear entre as variáveis restantes, que explique a maior quantidade de variância restante, e assim sucessivamente, até que seja explicada a variância total dos dados, sendo que no final deste procedimento ter-se-á fatores ortogonais (BODRUD-DOZA et al., 2016). Diante do exposto, este estudo teve como objetivo aplicar a análise fatorial e a análise de componentes principais, para buscar identificar as possíveis fontes de poluição do rio Tramandaí/RS.

\section{MATERIAIS E MÉTODOS}

\section{Caracterização da área}

A BH do Litoral é formada pelas bacias do Leste e do extremo Sul do Estado e é dividida em cinco bacias, sendo elas: Tramandaí, Litoral Médio, Camaquã, Mirim-São Gonçalo e Mampituba. Neste trabalho foram analisados os pontos: GER 15, GER 16, GER 17 e GER 18, (coordenadas geográficas na Tabela 1) localizado no rio Tramandaí e inserido na Bacia Hidrográfica Tramandaí dentro da Região da Bacia Litorânea (FEPAM, 2018).

Tabela 1: coordenadas geodésicas referentes aos pontos deste estudo.

\begin{tabular}{|l|l|l|}
\hline Pontos & Latitude & Longitude \\
\hline GER 15 & $-29^{\circ} 57^{\prime} 012^{\prime \prime}$ & $-50^{\circ} 09^{\prime} 052^{\prime \prime}$ \\
\hline GER 16 & $-29^{\circ} 58^{\prime} 010^{\prime \prime}$ & $-50^{\circ} 08^{\prime} 021^{\prime \prime}$ \\
\hline GER 17 & $-29^{\circ} 58^{\prime} 057^{\prime \prime}$ & $-50^{\circ} 08^{\prime} 03^{\prime \prime}$ \\
\hline GER 18 & $-29^{\circ} 58^{\prime} 037^{\prime \prime}$ & $-50^{\circ} 07^{\prime} 022^{\prime \prime}$ \\
\hline
\end{tabular}

A Bacia Hidrográfica do Rio Tramandaí encontra-se na porção nordeste do Estado do Rio Grande do Sul, apresenta uma área de $3.144,84 \mathrm{~km}^{2}$, que engloba os municípios de Capão da Canoa, Dom Pedro de Alcântara, Imbé, Maquiné, Osório, Torres Tramandaí e Três Forquilhas. Os principais usos de água desta bacia são para a irrigação e abastecimento público (SEMA, 2018; FEPAM, 2018).
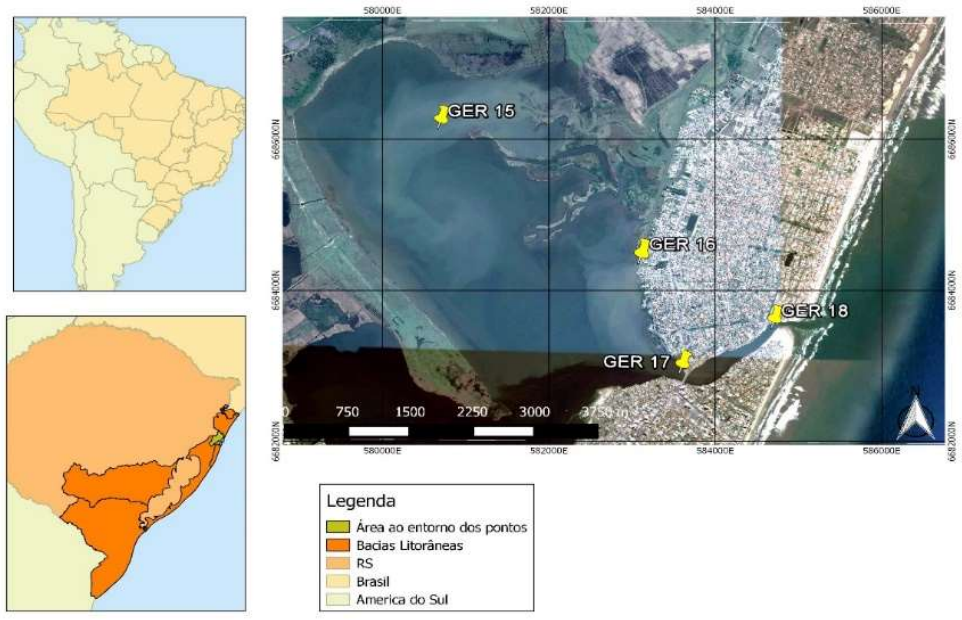

Figura 1: Mapa de localização da área deste estudo. 


\section{Compilação das Variáveis Limnológicas}

Foram utilizados dados secundários de qualidade da água disponibilizados pela Fundação Estadual de Proteção Ambiental Henrique Luis Roessler (FEPAM/RS), compreendidos entre os anos de 2005 a 2013. Sendo que as variáveis escolhidas por apresentar um menor percentual de falhas amostrais foram: Oxigênio Dissolvido (OD), Coliformes Termotolerantes (CT), Potencial Hidrogeniônico (pH), Demanda Bioquímica de Oxigênio $\left(\mathrm{DBO}_{5}\right)$, Cloretos $\left(\mathrm{Cl}^{-}\right)$, Nitrogênio Amoniacal $\left(\mathrm{NH}_{3}\right)$, Nitrogênio Total (NT), Fósforo Total (PT), Condutividade Elétrica (CE), Turbidez (TH), Sólidos Totais (ST), Temperatura da Água ( $\mathrm{T}_{\mathrm{H} 2 \mathrm{O}}$ ) e Temperatura do $\operatorname{Ar}\left(T_{A r}\right)$ As coletas foram realizadas pela FEPAM de acordo com a metodologia descrita por CETESB (1987). Os métodos analíticos bem como a preservação das amostras seguem os procedimentos definidos por APHA (2005).

\section{Análise estatística dos dados}

Primeiramente normalizou-se os dados de qualidade da água, posteriormente construiu-se uma matriz de dados expressos por $X=(x i, j)$, em que $i=1 . . n$ amostragens $(72)$ e $j=1 \ldots$ variáveis (13). Em seguida, transformou-se a matriz de dados originais em uma matriz de correlações [R] (pxp), sendo que " $p$ " corresponde as variáveis de qualidade da água.

Através da AF/ACP se transformou o conjunto original das variáveis observadas em um novo conjunto de variáveis, denominadas componentes principais (CP). Segundo Hair et al. (2009), as três primeiras componentes, devem explicar o máximo de variabilidade total dos dados, sendo que a primeira não esteja correlacionada com a segunda e a segunda não esteja correlacionada com a terceira, e esta não esteja correlacionada nem com a primeira nem com a segunda, e assim sucessivamente, até que as CPs expliquem mais que $70 \%$ da variância total dos dados. Para a para a realização dos cálculos fez-se uso do software $R$ versão 3.4.3.

\section{RESULTADOS E DISCUSSÃO}

\section{Análise de Componentes Principais (ACP)/Análise Fatorial (AF)}

As variáveis $\mathrm{CE}, \mathrm{ST}, \mathrm{TH}, \mathrm{OD}, \mathrm{T}_{\mathrm{H} 2 \mathrm{O}}, \mathrm{T}_{\mathrm{AR}}$ e $\mathrm{ST}$ apresentaram maiores coeficientes de correlação (Tabela

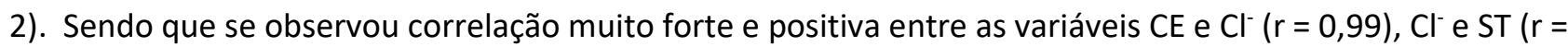
0,98), CE e ST $(r=0,98)$, e $\mathrm{T}_{\mathrm{H} 2 \mathrm{O}}$ e $\mathrm{T}_{\mathrm{AR}}(\mathrm{r}=0,96)$. As correlações entre ST e $\mathrm{Cl}^{-}$eram esperadas, pois os $\mathrm{Cl}^{-}$podem estar relacionados as dissoluções de sais alterando também a CE. De acordo com Sperling (2005) quaisquer contaminantes predispostos nos corpos hídricos contribuem para o aumento dos ST. Por conseguinte, OD e $\mathrm{T}_{\mathrm{H} 2 \mathrm{O}}(\mathrm{r}=0,91)$, OD e TAR $(r=0,86)$, CE e TH $(r=0,76)$, $\mathrm{TH}^{-} \mathrm{Cl}^{-}(r=0,74)$ e ST e TH $(r=0,70)$, apresentaram correlações negativas significativa.

Segundo Libânio (2010) o aumento da temperatura, faz com que ocorra a redução da taxa de solubilidade dos gases, o que explica a relação inversa do OD com a temperatura da água. A relação entre OD com $\mathrm{Cl}^{-}, \mathrm{CE}$ estão relacionados com a forma como o constituinte responsável, pela presença dessas 
variáveis $\left(\mathrm{Cl}^{-}, \mathrm{CE}\right)$, se apresenta na água, ou seja, através sólidos dissolvidos (SD), por isso a medida que aumenta os SD, diminui os sólidos em suspensão (SS) que consequentemente é a forma dos constituintes responsável pela turbidez. O mesmo pode explicar os ST, uma vez que este é calculado pelo somatório dos SD e SS, então a medida que aumentar os SS, os ST devem diminuir para que a equação se mantenha em equilíbrio (BRAGA, 2015).

Tabela 2: Matriz de correlação do rio Tramandaí, contendo as 13 variáveis de qualidade da água.

\begin{tabular}{|l|l|l|l|l|l|l|l|l|l|l|l|l|l|}
\hline & $\mathrm{CL}-$ & $\mathrm{CT}$ & $\mathrm{CE}$ & $\mathrm{DBO}_{5}$ & $\mathrm{PT}$ & $\mathrm{NH}_{3}$ & $\mathrm{NT}$ & $\mathrm{OD}$ & $\mathrm{pH}$ & $\mathrm{ST}$ & $\mathrm{T}_{\mathrm{H} 2 \mathrm{O}}$ & $\mathrm{T}_{\mathrm{AR}}$ & $\mathrm{TH}$ \\
\hline $\mathrm{Cl}^{-}$ & 1,00 & & & & & & & & & & & & \\
\hline $\mathrm{CT}$ & $-0,05$ & 1,00 & & & & & & & & & & \\
\hline $\mathrm{CE}$ & 0,99 & $-0,05$ & 1,00 & & & & & & & & & \\
\hline $\mathrm{DBO}_{5}$ & $-0,26$ & $-0,12$ & $-0,29$ & 1,00 & & & & & & & & & \\
\hline $\mathrm{PT}$ & $-0,07$ & $-0,04$ & $-0,07$ & 0,33 & 1,00 & & & & & & & & \\
\hline $\mathrm{NH}_{3}$ & $-0,12$ & 0,51 & $-0,16$ & 0,12 & 0,21 & 1,00 & & & & & & & \\
\hline $\mathrm{NT}$ & $-0,13$ & $-0,03$ & $-0,11$ & $-0,12$ & $-0,03$ & $-0,16$ & 1,00 & & & & & \\
\hline $\mathrm{OD}$ & 0,41 & 0,06 & 0,35 & 0,53 & 0,43 & $-0,07$ & $-0,24$ & 1,00 & & & & & \\
\hline $\mathrm{pH}$ & 0,17 & 0,22 & 0,16 & $-0,22$ & $-0,45$ & 0,01 & 0,13 & $-0,18$ & 1,00 & & & & \\
\hline $\mathrm{ST}$ & 0,98 & $-0,07$ & 0,98 & $-0,28$ & 0,00 & $-0,11$ & $-0,11$ & 0,36 & 0,14 & 1,00 & & & \\
\hline $\mathrm{T}_{\mathrm{H} 2 \mathrm{O}}$ & $-0,42$ & $-0,25$ & $-0,37$ & $-0,51$ & $-0,38$ & $-0,09$ & 0,22 & $-0,91$ & 0,32 & $-0,36$ & 1,00 & & \\
\hline $\mathrm{T}_{\mathrm{AR}}$ & $-0,48$ & $-0,34$ & $-0,42$ & $-0,41$ & $-0,30$ & $-0,16$ & 0,28 & $-0,86$ & 0,20 & $-0,41$ & 0,96 & 1,00 & \\
\hline $\mathrm{TH}$ & $-0,74$ & 0,22 & $-0,76$ & 0,55 & 0,40 & 0,32 & $-0,18$ & 0,13 & $-0,16$ & $-0,70$ & $-0,04$ & 0,01 & 1,00 \\
\hline
\end{tabular}

$\mathrm{Cl}^{\prime}$ : Cloretos; CT: Coliformes Termotolerantes; CE: Condutividade Elétrica; $\mathrm{DBO}_{5}$ : Demanda Bioquímica de Oxigênio; PT: Fósforo Total; $\mathrm{NH}_{3}$ : Nitrogênio Amoniacal; NT: Nitrogênio Total; OD: Oxigênio Dissolvido; pH: Potencial Hidrogeniônico; ST: Sólidos Totais; $\mathrm{T}_{\mathrm{H} 2 \mathrm{O}}$ : Temperatura da Água; $\mathrm{T}_{\mathrm{AR}}$ : Temperatura do Ar e TH: Turbidez.

Segundo Goméz (2013) e Olsen et al. (2012), a seleção do número de componentes está fundamentada na porcentagem acumulada da variância total que deve ser acima de $70 \%$ para proporcionar uma noção aceitável do que realmente está ocorrendo no manancial estudado, ou seja, representando assim a variância original. Neste estudo conforme a Tabela 3, podemos observar que os dois primeiros fatores explicaram mais de $70 \%$ da variância total dos dados, sendo, então utilizados para explicar as principais fontes de poluição existentes no rio Tramandaí.

Tabela 3: Fatores encontrados e a explicação da variância da amostra.

\begin{tabular}{|l|l|l|}
\hline Fator & Variância Total Explicada (\%) & Variância Total Acumulada (\%) \\
\hline 1 & 60,86 & 60,86 \\
\hline 2 & 12,74 & 73,60 \\
\hline
\end{tabular}

Através da $\mathrm{AF} / \mathrm{ACP}$ gerou-se as componentes principais (CPs) que expressam a relação entre fatores e variáveis e segundo Ferreira (2010) permitem identificar as variáveis com maiores interações em cada componente conforme a Tabela 4. A primeira componente explica $60,86 \%$ da variabilidade total dos dados, sendo representada pelas variáveis $\mathrm{Cl}^{\prime}, \mathrm{CT}, \mathrm{NH}_{3}, \mathrm{OD}, \mathrm{ST}, \mathrm{TH}_{2} \mathrm{O}$ e $\mathrm{T}_{\mathrm{AR}}$, e estão associadas, quando relacionadas aos impactos antrópicos, as variáveis indicativas de cargas de nutrientes provenientes de esgotos domésticos, fertilizantes orgânicos e excremento de animais, oriundos de atividades pecuárias, e a presença do $\mathrm{NH}_{3}$ indica que as fontes de poluição associadas a $\mathrm{CP}_{1}$ são recentes (SPERLING, 2007). Já a $\mathrm{CP}_{2}$ explica 12,74\% da variância total, sendo composta por $\mathrm{CE}, \mathrm{DBO}_{5}, \mathrm{PT}, \mathrm{NT}$, pH e TH, e possivelmente está relacionada com irrigação mal projetada, utilização inadequada de fertilizantes químicos e pesticidas (NUVOLARI, 2013).

Ouyang (2005) em seu estudo aplicou a análise de componentes principais e a análise fatorial para avaliar a qualidade da água de um rio da Florida nos Estados Unidos, para identificar os parâmetros mais 
relevantes sendo estes: carbono orgânico total, oxigênio dissolvido, nitrogênio total, nitrato e nitrito, fósforo total, alcalinidade, salinidade, Mg e Ca.

Tabela 4: Fatores encontrados e a explicação da variância da amostra.

\begin{tabular}{|l|l|l|}
\hline Variáveis & $\mathbf{C P}_{1}$ & $\mathbf{C P}_{\mathbf{2}}$ \\
\hline $\mathrm{Cl}^{-}$ & 0,91 & $-0,38$ \\
\hline $\mathrm{CT}$ & 0,88 & $-0,43$ \\
\hline $\mathrm{CE}$ & 0,05 & 0,73 \\
\hline $\mathrm{DBO}$ & 0,04 & 0,77 \\
\hline $\mathrm{PT}$ & 0,15 & 0,71 \\
\hline $\mathrm{NH} 3$ & $-0,78$ & $-0,54$ \\
\hline $\mathrm{NT}$ & $-0,22$ & $-0,76$ \\
\hline $\mathrm{OD}$ & 0,71 & 0,58 \\
\hline $\mathrm{pH}$ & $-0,03$ & $-0,76$ \\
\hline $\mathrm{ST}$ & 0,87 & $-0,39$ \\
\hline $\mathrm{T}_{\text {H2O }}$ & $-0,75$ & $-0,61$ \\
\hline $\mathrm{T}_{\mathrm{AR}}$ & $-0,82$ & 0,35 \\
\hline $\mathrm{TH}$ & $-0,51$ & $\mathbf{0 , 7 7}$ \\
\hline
\end{tabular}

$\mathrm{Cl}^{\prime}$ : Cloretos; $\mathrm{CT}$ : Coliformes Termotolerantes; $\mathrm{CE}$ : Condutividade Elétrica; $\mathrm{DBO}_{5}{ }^{20}$ : Demanda Bioquímica de Oxigênio; PT: Fósforo Total; $\mathrm{NH}_{3}$ : Nitrogênio Amoniacal; NT: Nitrogênio Total; OD: Oxigênio Dissolvido; pH: Potencial Hidrogeniônico; ST: Sólidos Totais; $\mathrm{TH}_{2} \mathrm{O}$ : Temperatura da Água; $\mathrm{T}_{\mathrm{AR}}$ : Temperatura do Ar e TH: Turbidez.

\section{CONCLUSÕES}

Através deste estudo foi possível concluir que o rio Tramandaí apresenta basicamente duas fontes de poluição. Bem como que a estatística multivariada através da análise fatorial/análise de componentes principais são ferramentas que pode ser utilizada para fins de monitoramento da qualidade da água quando se busca investimentos de baixo custo com resultados confiáveis e rápidos.

AGRADECIMENTOS: Agradecemos a CAPES (Coordenação de Aperfeiçoamento de Pessoal de Nível Superior) pela concessão da bolsa, durante todo esse período de realização do Doutorado, que está sendo realizado no PPG em Recursos Hídricos, e a FEPAM (Fundação Estadual de Proteção Ambiental Henrique Roessler), por disponibilizar os dados para o desenvolvimento desta pesquisa.

\section{REFERÊNCIAS}

APHA. American Public Health Association. Standard methods for the examination of water and wastewater. 21 ed. Washington: American Public Health Association, APHA/AWWA/WEF, 2005.

BADILLO-CAMACHO, J.; REYNAGA-DELGADO, E.; BARCELOQUINTAL, I.; DEL VALLE, P. F. Z.; LÓPEZ-CHUKEN, U. J.; OROZCO-GUAREÑO, E.; BOBADILLA, J. I. A.; GOMEZSALAZAR, S.. Water quality assessment of a Tropical Mexican lake using multivariate statistical techniques. Journal of Environmental Protection, v.6, n.3, p.215-224, 2015. DOI: http://dx.doi.org/10.4236/jep.2015.63022

BERTOSSI, A. P. A.; CECÍlIO, R. A.; NEVES, M. A.; GARCIA, G.. $O$. Qualidade da água em microbacias hidrográficas com diferentes coberturas do solo no sul do Espírito Santo. Revista Árvore, v.37, n.1, p.107-117, 2013.

BODRUD-DOZA, M. D.; ISLAM, A. R. M. T.; AHMED, F.; DAS, S.; SAHA, N.; RAHMAN, M. S.. Characterization of groundwater quality using water evaluation indices, multivariate statistics and geostatistics in central Bangladesh. Water Science, v.30, n.1, p.19-40, 2016. DOI: http://dx.doi.org/10.1016/j.wsj.2016.05.001

BRAGA, B.; TUNDISI, J. G; TUNDISI, T. M.; CIMINELLI, V. S. T.. Águas doces no Brasil: capital ecológico, uso e conservação. 4 ed. São Paulo: Escrituras, 2015.

CETESB. Companhia de Tecnologia de Saneamento Ambiental. Guia de coleta e preservação de amostras de água. São Paulo: CETESB, 1987.

CORRAR, L. J.; PAULO, E.; DIAS FILHO, J. M.. Análise multivariada para os cursos de administração, ciências contábeis e economia. 5 ed. São Paulo: Atlas, 2014. 344 p.

FERREIRA, D. F.. Estatística multivariada. 2 ed. Lavras: UFLA, 2011. 
FEPAM. Fundação Estadual de Proteção Ambiental Henrique Roessler. Monitoramento da qualidade da água da região hidrográfica das bacias litorâneas. 2018.

GOMES, A. I.; PIRES, J. C. M; FIGUEIREDO, S. A.; BOAVENTURA, R. A. R.. Optimization of River Water Quality Surveys by Multivariate Analysis of Physicochemical, Bacteriological and Ecotoxicological Data. Water Resources Management, v.28, n.5, p.1345-1361, 2014.

GOMÉZ, D. M.. Estudio del modelamiento de la calidad del agua del río Sinú, Colombia. Revista de Ingenierías: Universidad de Medellín, v.12, n.22, p.33-44, 2013.

HAIR, F. J.; BLACK, W. C.; BABIN, B. J.; ANDERSON, R. E.; TATHAM, R. L.. Análise multivariada de dados. 6 ed. Porto Alegre: Bookman, 2009.

HASHEMI, S.; JAVAHERIAN, A.;ATAEE-POUR, M.; TAHMASEBI, P.; KHOSHDEL, H.. Channel characterization using multiplepoint geostatistics, neural network, and modern analogy: a case study from a carbonate reservoir, southwest Iran. Journal of Applied Geophysics, v.111, p.47-58, 2014. DOI: http://dx.doi.org/10.1016/i.jappgeo.2014.09.015

HELLER, L.; PÁDUA, L.. Abastecimento de água para consumo humano. 2. ed. Belo Horizonte: UFMG, 2010.

LIBÂNIO, M.. Fundamentos de qualidade e tratamento de água. Campinas: Átomo, 2010.

MONICA, N.; CHOI, K.. Temporal and spatial analysis of water quality in Saemangeum watershed using multivariate statistical techniques. Paddy and Water Environment, v.14, n.1, p.3-17, 2015. DOI: http://dx.doi.org/10.1007/s10333$\underline{014-0475-6}$

NUVOLARI, A.. Esgoto sanitário: coleta, transporte, tratamento e reúso agrícola. 2 ed. São Paulo: Blucher, 2011.
OLSEN, R. L.; CHAPPELL, R. W.; LOFTIS, J. C.. Water quality sample collection, data treatment and results presentation for principal components analysis - literature review and Illinois River watershed case study. Water Research, v.46, n.9, p.3110-3122, 2012.

OUYANG, Y.. Evaluation of river water quality monitoring stations by principal component analysis. Water Research, v.39, n.12, p.2621-2635, 2005

SEMA. Secretaria do Ambiente e Desenvolvimento Sustentável do Rio Grande do Sul. Bacias Hidrográficas do RS. 2018 .

SPERLING, M. V.. Estudos e modelagem da qualidade da água de rios. Belo Horizonte: DESA/UFMG, 2007.

SPERLING, M. V.. Introdução à qualidade das águas e ao tratamento de esgotos. 3 ed. Belo Horizonte: DESA - UFMG, 2005.

VAROL, S.; DAVRAZ, A.. Evaluation of the groundwater quality with WQI (Water Quality Index) and multivariate analysis: a case study of the Tefenni plain (Burdur/Turkey). Environmental Earth Sciences, v.73, n.4, p.1725-1744, 2014. Springer Nature. DOI: http://dx.doi.org/10.1007/s12665014-3531-z

VICINI, L; SOUZA, A. M.. Análise multivariada da teoria à pratica. Santa Maria: Biblioteca Central da UFSM, 2005.

VOZA, D.; VUKOVIC, M.; TAKIC, L.; NIKOLIC, D.; MLADENOVIC-RANISAVLJEVIC, I.. Application of multivariate statistical techniques in the water quality assessment of Danube river, Serbia. Archives of Environmental Protection, v.41, n.4, p.96-103, 2015. DOI:

http://dx.doi.org/10.1515/aep-2015-0044

A CBPC - Companhia Brasileira de Produção Científica (CNPJ: 11.221.422/0001-03) detém os direitos materiais desta publicação. Os direitos referem-se à publicação do trabalho em qualquer parte do mundo, incluindo os direitos às renovações, expansões e disseminações da contribuição, bem como outros direitos subsidiários. Todos os trabalhos publicados eletronicamente poderão posteriormente ser publicados em coletâneas impressas sob coordenação da Sustenere Publishing, da Companhia Brasileira de Produção Científica e seus parceiros autorizados. Os (as) autores (as) preservam os direitos autorais, mas não têm permissão para a publicação da contribuição em outro meio, impresso ou digital, em português ou em tradução. 